\title{
Prenatal nicotine exposure induces gender-associated left ventricular-arterial uncoupling in adult offspring
}

\author{
ZHENHUA WANG ${ }^{1,2^{*}}$, QIUFANG OUYANG ${ }^{1,3^{*}}$, ZIYANG HUANG ${ }^{1}$, LING LIN $^{4}$, \\ ER'MEI YU $^{1}$ and MARKUS W. FERRARI ${ }^{5}$
}

\author{
${ }^{1}$ Cardiovascular Department, Second Affiliated Hospital and Second Clinical Medical College, \\ Fujian Medical University, Quanzhou, Fujian 362000; ${ }^{2}$ Quanzhou Medical College, Quanzhou, Fujian 362000; \\ ${ }^{3}$ Ultrasound Department, Second Affiliated People's Hospital, Fujian University of Traditional Chinese Medicine, \\ Fuzhou, Fujian 350003; ${ }^{4}$ Rheumatism Department, Second Affiliated Hospital and Second Clinical Medical College, \\ Fujian Medical University, Quanzhou, Fujian 362000, P.R. China; ${ }^{5}$ Clinic of Internal Medicine 1, HSK, \\ Clinic of the City of Wiesbaden and the HELIOS Group, Wiesbaden D-65199, Germany
}

Received February 15, 2014; Accepted December 19, 2014

DOI: $10.3892 / \mathrm{mmr} .2015 .3364$

\begin{abstract}
Alterations in vascular or myocardial structure and function have been demonstrated in offspring subjected to prenatal nicotine exposure (PNE), however, limited data are available on how these changes interact. The present study assessed the hypothesis that prenatal nicotine exposure induced gender-specific alterations of left ventricular-arterial coupling indices in adult offspring. Female Sprague-Dawley rats were exposed to either nicotine $(8 \mathrm{mg} / \mathrm{kg} / \mathrm{day})$ or saline via subcutaneous osmotic mini-pumps throughout gestation. Male and female offspring, aged 12 months, underwent non-invasive echocardiography and invasive left ventricular cannulation. Left ventricular-arterial coupling was analysed as the ratio of effective arterial elastance (Ea) to ventricular end-systolic elastance (Ees). The left ventricular myocardium and aorta were stained with hematoxylin and eosin and the myocardial cell cross-sectional area was calculated. Simultaneously, the ratio of medium thickness to internal diameter in the aorta and mesenteric artery was determined. The fibrosis component of left ventricle myocardium was analyzed by Sirius-red staining and further confirmed by hydroxyproline determination. The elastic properties of the aortic wall were analyzed by van Gieson staining. PNE caused significant increases in pulse pressure $(56.36 \pm 7.41$ vs.
\end{abstract}

Correspondence to: Professor Ziyang Huang, Cardiovascular Department, Second Affiliated Hospital and Second Clinical Medical College, Fujian Medical University, 34 Zhongshan North Road, Quanzhou, Fujian 362000, P.R. China

E-mail: huagzy@126.com

*Contributed equally

Key words: prenatal exposure, gender, nicotine, ventricular-arterial coupling, stiffness
$50.16 \pm 4.94 \mathrm{mmHg} ; \mathrm{P}<0.05)$ and left ventricular meridional wall stress (78.25 \pm 9.12 vs. $\left.69.64 \pm 7.58 \mathrm{kdyne} / \mathrm{cm}^{2} ; \mathrm{P}<0.05\right)$ in male offspring compared with the control. Conversely, no similar effect was observed in female offspring. An elevated augmentation index was noted in male and female pups. Additionally, Ea/Ees was reduced in PNE males compared with control males, due to a disproportionate increase in Ees vs. Ea whereas in females, Ea/Ees did not differ significantly due to tandem increase in Ea and Ees. In addition, collagen cross-linking was markedly higher in male offspring, whereas it was unaltered in females compared with their respective controls. Fragmentation of the elastic network in the aorta and the increased ratio of medial thickness to internal diameter in the mesenteric artery were more evident in male offspring when compared with female offspring. PNE caused combined ventricular-arterial stiffening in male and female offspring, with lower $\mathrm{Ea} / \mathrm{Ees}$ in males, while $\mathrm{Ea} / \mathrm{Ees}$ was preserved in females. Enhanced collagen cross-linking in the myocardium, underdeveloped elastic fibers in the aorta and remodeled resistance vessels were associated with pathological ventricular arterial mismatching. The results of the present study indicated that male offspring were more susceptible to the development of ventricular and arterial dysfunction in response to PNE compared with female offspring.

\section{Introduction}

Nicotine is a major component of cigarette smoke. The adverse effects of nicotine on the cardiovascular system have been well documented (1,2). A growing body of evidence has revealed that offspring exposed to an adverse intrauterine environment develop a series of cardiovascular disorders, including atherosclerosis (3), obesity (4), cardiac arrhythmias (5), coronary artery disease (6) and hypertension $(7,8)$. In addition, compelling evidence has demonstrated that acute and chronic cigarette smoking exacerbates arterial stiffness (9), as well as myocardial fibrosis (10). In canine models exposed 
to nicotine, increased left ventricle (LV) chamber stiffness is identified due to increased collagen deposition and collagen cross-links in the myocardium (11). However, insufficient data are available on the effects of prenatal nicotine exposure (PNE) on combined ventricular-arterial stiffening, designated 'ventricular-arterial uncoupling'.

Ventricular-arterial coupling, meaning the interaction of the heart with the systemic vasculature, is a key determinant of cardiovascular performance. Ventricular-arterial integration can be quantified via examination of the ratio of effective arterial elastance (Ea) and LV end-systolic elastance (Ees). $\mathrm{Ea}$, the ratio of end systolic pressure (PES)/stroke volume, is representative of arterial loading, while Ees is an indicator of ventricular end-systolic elastance. The ratio is used to index relative coupling between the heart and vascular system (12).

Boychuk et al (13) reported that PNE gender-dependently compromised cardiorespiratory integration in vivo during early postnatal development and primarily affected male offspring. Clinically, convincing evidence has indicated that smoking disrupts complex hemodynamic mechanisms even in young smokers with a resultant increase in myocardial workload, a decreased capacity for coronary perfusion and blunted ventricular-vascular dynamics (14). However, to date, few animal studies have been designed to investigate the effects of PNE on ventricular-vascular integration in adult offspring in vivo. Thus, the aim of the present study was to examine whether PNE causes a similar alteration in ventricular-arterial coupling and subsequently to investigate whether this is associated with myocardial fibrosis, aortic elasticity properties and the morphology of resistance vessels.

\section{Materials and methods}

Ethics statement. All the procedures and protocols were approved by the Fujian Medical University Institutional Animal Care and Use Committee (Fuzhou, China) and followed the guidelines outlined by the National Institutes of Health Guide for the Care and Use of Laboratory Animals (National Institutes of Health, Bethesda, MD, USA).

Animals and experimental protocol. Female Sprague-Dawley rats $(n=10)$ weighing $300 \pm 35 \mathrm{~g}$ were purchased from the Shanghai Laboratory Animal Center of the Chinese Academy of Sciences (Shanghai, China). The rats were exposed to nicotine $(\mathrm{n}=8,8 \mathrm{mg} / \mathrm{kg} /$ day $)$ or saline $(\mathrm{n}=4)$ via subcutaneous osmotic mini-pumps (Alzct Model 2ML4; Alza Corp., Palo Alto, CA, USA) throughout gestation as described previously $(8,15)$. Natal pups were kept with their mothers until weaning. At weaning, male and female pups were separated and housed in temperature and humidity-controlled rooms with a 12 h light-dark cycle. They were administered standard chow and mineral water ad libitum. Caudal artery systolic blood pressure (SBP), diastolic blood pressure (DBP) and pulse pressure (PP) was monitored in nonanesthetized pups every 2 weeks with a tail cuff system (BP-98A; Softron, Tokyo, Japan). Male and female offspring were sacrificed by intraperitoneal injection of $200 \mathrm{mg} / \mathrm{kg}$ pentobarbital (Sigma-Aldrich, St. Louis, MO, USA) at 12 months-old to determine the effects of PNE on the coupling conditions of the LV and artery system.

Quantification of cardiac geometry and function using echocardiography. Cardiac geometry and function of anesthetized offspring (ketamine $50 \mathrm{mg} / \mathrm{kg}$ and diazepam $2.5 \mathrm{mg} / \mathrm{kg}$ ) were evaluated by transthoracic echocardiography (Sonos 7500; Philips Healthcare, Eindhoven, Netherlands) with a $15 \mathrm{MHz}$-transducer. The dosage regimen was previously demonstrated to have minimal cardiorespiratory effects when compared with other suitable anesthetics (16). For calculation of intraobserver variability, examinations were repeated by the same examiner and for interobserver variability, examinations were performed independently by two investigators. The probe was placed to obtain short and long-axis and four-chamber views. From the long-axis view, an $\mathrm{M}$ mode trace of the LV was obtained, and left ventricular end systolic diameter, LV end diastolic diameter and LV wall thickness were measured. Left ventricular end diastolic volume (EDV) and end systolic volume were determined using the biplane Simpson method (17). Stroke volume was the difference between end-diastolic volume and end-systolic volume. LV end-systolic meridional wall stress was calculated using the following formula $(18,19)$ : $0.34 \mathrm{PD} /[(1+\mathrm{h} / \mathrm{D}) \mathrm{h}]$, where $\mathrm{P}$ is $\mathrm{LV}$ pressure $(\mathrm{mmHg}), \mathrm{D}$ is $\mathrm{LV}$ cavity diameter, $\mathrm{h}$ is wall thickness and 0.34 is the conversion factor from $\mathrm{mmHg}$ to gram-force $/ \mathrm{cm}^{2}$.

Assessment of left ventricular and arterial hemodynamics with a conductance catheter. Following the echocardiography study, a $1.5 \mathrm{~F}$ high-fidelity manometer-tipped catheter (SPR-407; Millar Instruments, Houston, TX, USA) was introduced through the right carotid artery into the left ventricle. PES and the maximal rates of increases and decreases in $\mathrm{LV}$ pressure $\left(\mathrm{dP} / \mathrm{dt}_{\max }\right.$ and $\mathrm{dP} / \mathrm{dt}_{\min }$, respectively) were recorded and analyzed using PowerLab Chart 4.1.2 software (ADInstruments, Inc., Bella Vista, New South Wales, Australia). Successively, central aortic SBP, DBP and PP were recorded when the conductance catheter was withdrawn from the LV into the ascending aorta. To quantitate the ventricular-arterial interaction, the LV and the arterial system are considered elastic chambers with known LV Ees and Ea, respectively. Effective pulmonary arterial elastance, as a measure of right ventricular (RV) afterload, was calculated as end-systolic pressure/stroke volume. An indicator of ventricular end-systolic elastance can be determined according to the following formula (20): Ees=0.10e $\mathrm{e}^{0.15 / \mathrm{EDVxdP} / \mathrm{dtmax}}$. Subsequently, the coupling parameter Ea/Ees was examined. The augmentation index (AI) was calculated as the ratio of $\Delta \mathrm{P}$ to $\mathrm{PP}$, where $\Delta \mathrm{P}$ was defined as the height from the shoulder of the reflected wave to the systolic peak (P2-P1) (21). For each rat, all the above-mentioned parameters were determined in 100-150 consecutive cardiac cycles and the results were averaged.

Elastic properties of the aorta. Following in vivo measurement of mechanical parameters, a $5 \mathrm{~mm}$ sample of the descending aorta and left ventricle was rapidly harvested. Fragmentation of the medial elastic fiber network (excluding the external and internal laminae) was evaluated on $6 \mu \mathrm{m}$ thick sections stained with van Gieson's solution by measuring the increase 
Table I. Body weight and basic caudal artery hemodynamic parameters.

\begin{tabular}{|c|c|c|c|c|}
\hline \multirow[b]{2}{*}{ Parameters } & \multicolumn{2}{|c|}{ Female offspring } & \multicolumn{2}{|c|}{ Male offspring } \\
\hline & Control $(n=9)$ & $\operatorname{PNE}(n=12)$ & Control $(n=10)$ & $\operatorname{PNE}(n=8)$ \\
\hline BW (g) & $348.17 \pm 21.42$ & $362.87 \pm 17.31$ & $628.58 \pm 26.01$ & $595.43 \pm 20.36$ \\
\hline LV/BW (mg/g) & $3.10 \pm 0.43$ & $2.91 \pm 0.53$ & $2.54 \pm 0.34$ & $2.42 \pm 0.44$ \\
\hline Heart rate (beats/min) & $394 \pm 17$ & $425 \pm 10$ & $410 \pm 15$ & $430 \pm 12$ \\
\hline SBPc (mmHg) & $141.95 \pm 5.16$ & $151.49 \pm 9.48$ & $148.41 \pm 7.14$ & $155.31 \pm 10.27$ \\
\hline DBPc (mmHg) & $89.37 \pm 4.26$ & $92.33 \pm 6.28$ & $98.42 \pm 9.84$ & $97.31 \pm 7.06$ \\
\hline $\mathrm{PPc}(\mathrm{mmHg})$ & $47.77 \pm 3.47$ & $53.28 \pm 6.36$ & $50.16 \pm 4.94$ & $56.36 \pm 7.41^{\mathrm{a}}$ \\
\hline
\end{tabular}

${ }^{a} \mathrm{P}<0.05$, versus gender-matched control. The results are expressed as the mean \pm standard deviation. BW, body weight; $\mathrm{LV}$, left ventricle; SBPc, caudal arterial systolic pressure; DBPc, caudal arterial diastolic pressure; PPc, caudal arterial pulse pressure; PNE, prenatal nicotine exposure.

in the number of elastic lamellae (22). The number of elastic lamellae in each of four quadrants were counted using light microscopy (BX51; Olympus Corp., Tokyo, Japan).

\section{Medial thickness to internal diameter ratio in the descending aorta and mesenteric artery. The aorta and mesenteric artery were stained with hematoxylin \& eosin (H\&E) and the aortic medium thickness (MT), internal diameter (ID) and the ratio of MT/ID were determined.}

Myocardial cell cross-sectional area and collagen volume fraction. Quantitative histomorphometry of the left ventricular myocardium was performed following H\&E staining. The mean of the cardiomyocyte cross-sectional area and the diameter were calculated using Image-Pro Plus 6.0 software (Media Cybernetics, Rockville, MD, USA). Collagen volume fraction was assessed using Sirius-red staining.

Collagen composition. The LV collagen content was estimated from the hydroxyproline content determined by the colorimetric method described by Leipner et al (23). The quantity of hydroxyproline was multiplied by the conversion factor 7.46 to calculate total collagen (24). To determine soluble collagen content, myocardium was extracted and digested with cyanogen bromide $(\mathrm{CNBr})$ according to the modified procedure of Yamamoto et al (25). The quantity of non-cross-linked (soluble) and cross-linked (insoluble) collagen in the myocardium was determined from the product of the percentage of collagen soluble to $\mathrm{CNBr}$ digestion, the total myocardial collagen concentration and the difference between the total collagen concentration and soluble collagen concentration, respectively. The association between insoluble and total collagen was used as an index of the degree of collagen cross-linking.

Statistical analysis. All values are expressed as the mean \pm standard deviation unless otherwise indicated. Differences were determined by unpaired Student's t-test. Intraobserver reproducibility was assessed by calculating the coefficient of variation and interobserver reproducibility by two-way analysis of variance. All reported probability values are two-tailed and $\mathrm{P}<0.05$ was considered to indicate a statistically significant difference. Statistical analyses were performed using SPSS 17.0 (SPSS, Inc., Chicago, IL, USA).

\section{Results}

Basic hemodynamic parameters. Maternal treatment with nicotine modified neither body weight (BW) nor LV/BW in either gender group (Table I). Similarly, maternal nicotine administration failed to affect SBP and DBP in the two groups, although the SBP in the male offspring tended to increase. However, caudal artery PP markedly increased in 12 month-old males, while it remained comparable in females compared with their gender-matched controls.

Echocardiographic properties. Echocardiographic examination revealed comparable LV end-systolic diameter, end-diastolic diameter and LV wall thickness (posterior and septal wall diastolic thickness) among the groups (Table II; Fig. 1). Prenatal nicotine exposure markedly reduced stroke volume by $25.71 \%$ in 12 month-old male offspring, but had no significant effect on stroke volume in female pups. Estimated left ventricular meridional wall stress was significantly enhanced in males whereas it remained unaltered in females following maternal nicotine administration. The intraobserver $(3.05 \%)$ and interobserver $(6.13 \%)$ variabilities were acceptable.

Ventricular and arterial hemodynamics. As shown in Table III, SBP, DBP and PP in males and females tended to be higher compared with age and gender-matched controls, however, no statistical significance was noted. Conversely, increased AI and $\mathrm{dP} / \mathrm{dt}_{\max }$ and decreased $\mathrm{dP} / \mathrm{dt}_{\text {min }}$ were found in males and females following maternal nicotine treatment compared with their respective control. These effects were more pronounced in males than in females. Ea/Ees did not differ significantly between PNE and control females due to tandem increases in Ea and Ees, suggesting that ventricular-arterial coupling was matched. By contrast, Ea/Ees was evidently lower in PNE males than control males $(0.77 \pm 0.04$ vs. $0.30 \pm 0.09 ; \mathrm{P}<0.05)$, owing to a disproportionate increase in Ees (by 2.19 -fold) vs. Ea (by 1.40 -fold).

Left ventricular cardiomyocyte cross-sectional area and total and insoluble collagen content. Maternal nicotine 
Table II. Echocardiographic properties.

\begin{tabular}{|c|c|c|c|c|}
\hline \multirow[b]{2}{*}{ Properties } & \multicolumn{2}{|c|}{ Female offspring } & \multicolumn{2}{|c|}{ Male offspring } \\
\hline & Control (n=9) & $\operatorname{PNE}(n=12)$ & Control $(n=10)$ & $\operatorname{PNE}(n=8)$ \\
\hline SWTd $(\mathrm{cm})$ & $0.16 \pm 0.03$ & $0.15 \pm 0.05$ & $0.23 \pm 0.04$ & $0.24 \pm 0.03$ \\
\hline PWTd (cm) & $0.19 \pm 0.04$ & $0.20 \pm 0.03$ & $0.26 \pm 0.06$ & $0.25 \pm 0.05$ \\
\hline $\operatorname{EDD}(\mathrm{cm})$ & $0.85 \pm 0.11$ & $0.80 \pm 0.09$ & $0.82 \pm 0.13$ & $0.72 \pm 0.14$ \\
\hline $\operatorname{ESD}(\mathrm{cm})$ & $0.59 \pm 0.10$ & $0.61 \pm 0.07$ & $0.63 \pm 0.10$ & $0.52 \pm 0.08$ \\
\hline $\mathrm{EDV}(\mathrm{ml})$ & $0.65 \pm 0.13$ & $0.53 \pm 0.14$ & $0.59 \pm 0.09$ & $0.37 \pm 0.11$ \\
\hline $\mathrm{SV}(\mathrm{ml})$ & $0.33 \pm 0.02$ & $0.32 \pm 0.03$ & $0.35 \pm 0.03$ & $0.26 \pm 0.03^{\mathrm{a}}$ \\
\hline LVMWS (kdyne/cm²) & $62.62 \pm 7.34$ & $64.92 \pm 9.64$ & $69.64 \pm 7.58$ & $78.25 \pm 9.12^{\mathrm{a}}$ \\
\hline
\end{tabular}

${ }^{a} \mathrm{P}<0.05$, versus gender-matched control. The results are expressed as the mean \pm standard deviation. PWTd, posterior wall diastolic thickness; SWTd, septal wall diastolic thickness; EDD, end diastolic diameter; ESD, end systolic diameter; EDV, end diastolic volume; SV, stroke volume; LVMWS, left ventricular meridional wall stress; PNE, prenatal nicotine exposure.
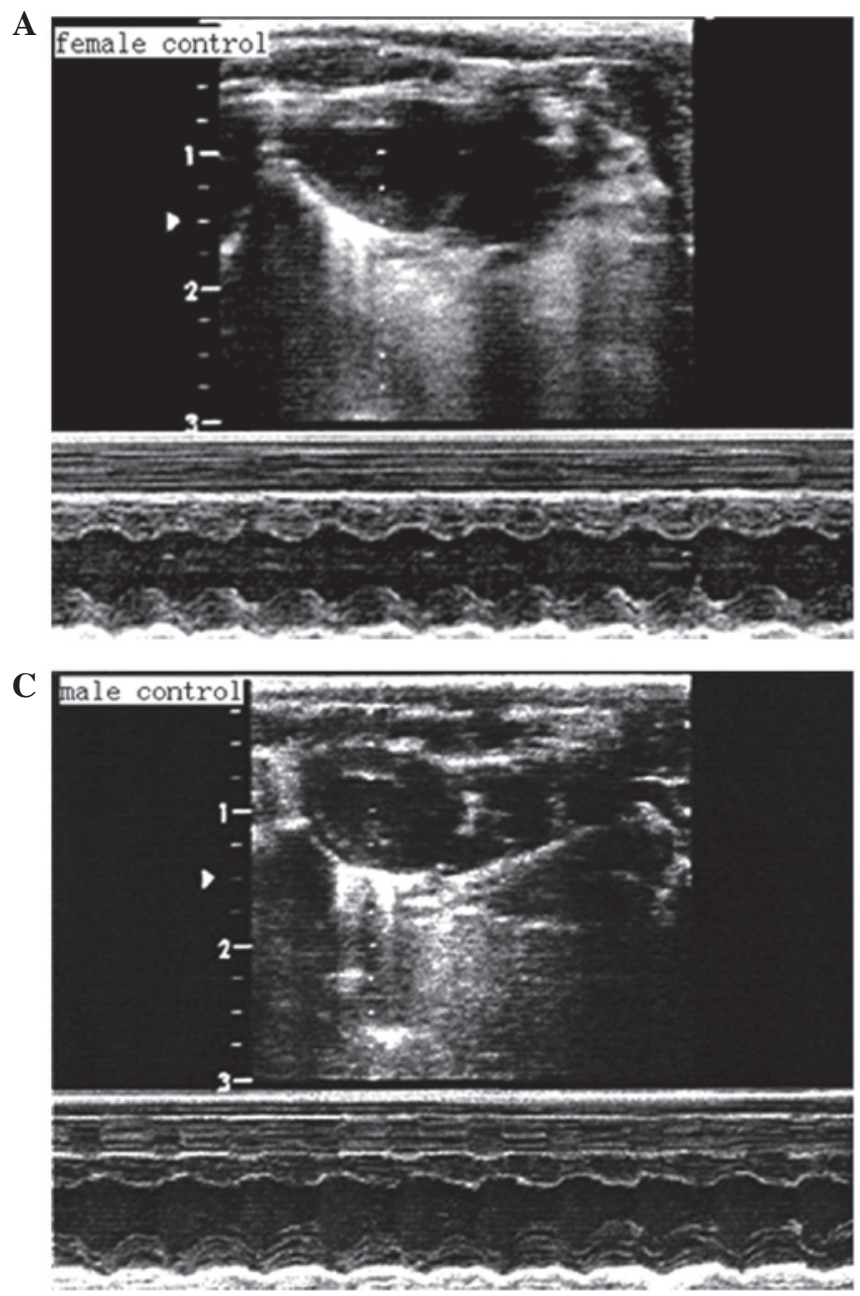
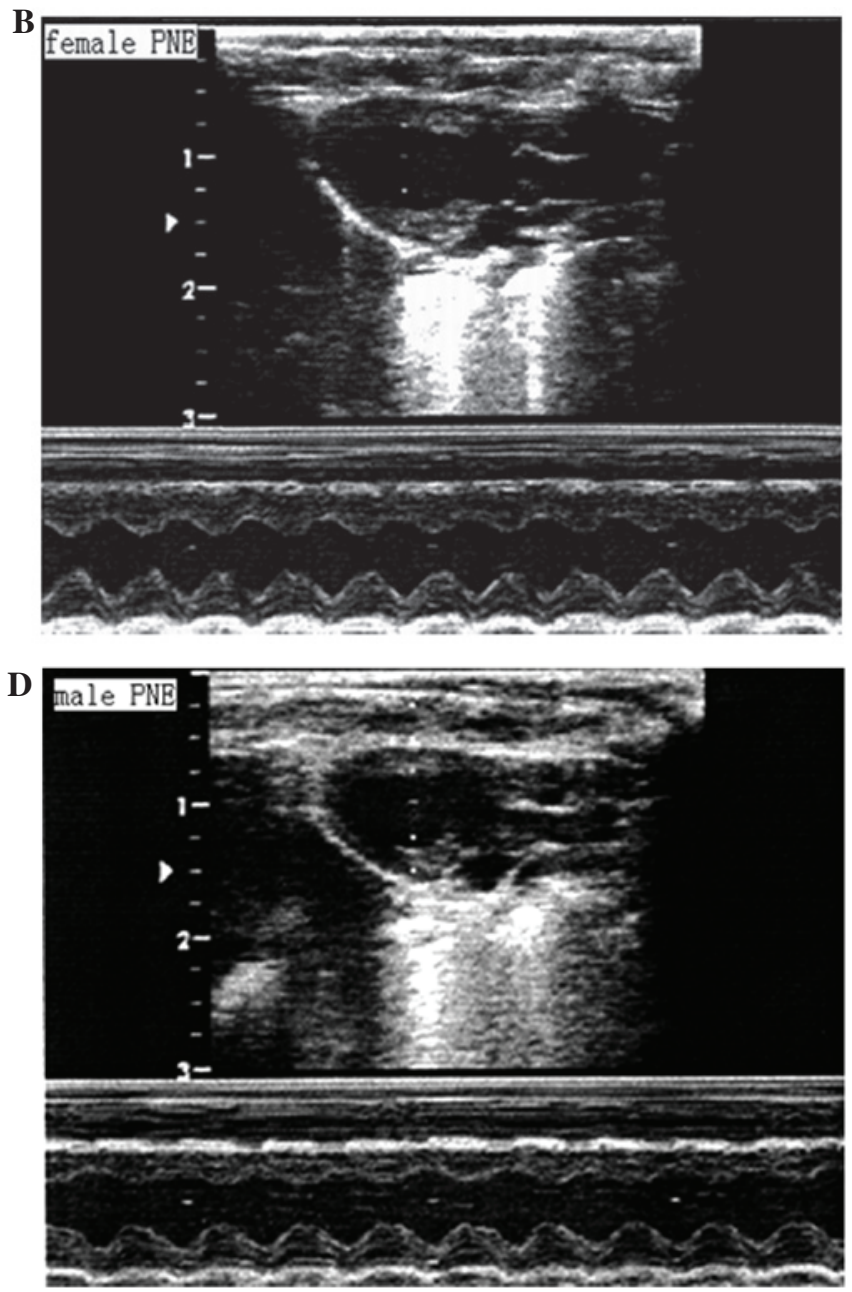

Figure 1. Representative M-mode echocardiogram in offspring exposed to vehicle or nicotine. Female offspring of (A) maternal saline exposure or (B) maternal PNE; and male offspring of (C) maternal saline exposure or (D) maternal PNE. Left ventricle chamber diameter and wall thickness differed insignificantly from their respective controls. PNE, prenatal nicotine exposure.

administration showed no significant affect of LV collagen content, as evidenced by similar collagen volume fractions and hydroxyproline quantification in the two groups (Fig. 2A-D). However, insoluble collagen (collagen cross-linking) content was markedly higher in male offspring $(51.64 \pm 6.83$ vs. $59.68 \pm 7.41 \mu \mathrm{g} / \mathrm{g} ; \mathrm{P}<0.05 ;$ Fig. 2E), whereas it exhibited only a mild but not significant increment in females compared with their controls (from an average of 50.74-54.81\%; P>0.05). In 
Table III. Hemodynamic parameters.

\begin{tabular}{|c|c|c|c|c|}
\hline \multirow[b]{2}{*}{ Hemodynamic parameters } & \multicolumn{2}{|c|}{ Female offspring } & \multicolumn{2}{|c|}{ Male offspring } \\
\hline & Control $(n=9)$ & $\operatorname{PNE}(n=12)$ & Control $(n=10)$ & $\operatorname{PNE}(n=8)$ \\
\hline Central SBP (mmHg) & $132.05 \pm 12.36$ & $145.37 \pm 14.91$ & $147.87 \pm 12.15$ & $155.47 \pm 17.06$ \\
\hline Central DBP (mmHg) & $92.07 \pm 8.15$ & $100.38 \pm 7.30$ & $105.26 \pm 9.13$ & $112.04 \pm 10.25$ \\
\hline $\mathrm{PP}(\mathrm{mmHg})$ & $32.47 \pm 3.11$ & $40.38 \pm 6.07$ & $41.42 \pm 4.06$ & $47.29 \pm 6.03^{*}$ \\
\hline Augmentation index $(\%)$ & $27.64 \pm 3.05$ & $34.46 \pm 4.25^{\mathrm{a}}$ & $29.12 \pm 5.21$ & $37.34 \pm 5.72^{\mathrm{a}}$ \\
\hline $\mathrm{Ea}(\mathrm{mmHg} / \mu \mathrm{l})$ & $0.35 \pm 0.09$ & $0.42 \pm 0.07^{\mathrm{a}}$ & $0.40 \pm 0.08$ & $0.56 \pm 0.10^{\mathrm{a}}$ \\
\hline $\mathrm{dP} / \mathrm{dt}_{\max }(\mathrm{mmHg} / \mathrm{ms})$ & $5694.03 \pm 312.47$ & $6624.38 \pm 483.15^{\mathrm{a}}$ & $6490.25 \pm 547.86$ & $7295.36 \pm 634.03^{\mathrm{a}}$ \\
\hline $\mathrm{dP} / \mathrm{dt}_{\min }(\mathrm{mmHg} / \mathrm{ms})$ & $4384.82 \pm 596.36$ & $3852.27 \pm 302.89^{\mathrm{a}}$ & $4918.75 \pm 368.25$ & $3156.89 \pm 248.26^{b}$ \\
\hline Ees $(\mathrm{mmHg} / \mu \mathrm{l})$ & $0.37 \pm 0.15$ & $0.65 \pm 0.13^{\mathrm{a}}$ & $0.53 \pm 0.09$ & $1.92 \pm 0.15^{\mathrm{b}}$ \\
\hline $\mathrm{Ea} / \mathrm{Ees}$ & $0.94 \pm 0.07$ & $0.65 \pm 0.05$ & $0.77 \pm 0.04$ & $0.30 \pm 0.09^{b}$ \\
\hline
\end{tabular}

${ }^{\mathrm{a}} \mathrm{P}<0.05$, versus gender-matched control; ${ }^{\mathrm{b}} \mathrm{P}<0.01$, versus gender-matched control. The results are expressed as the mean \pm standard deviation. SBP, systolic blood pressure; DBP, diastolic blood pressure; PP, pulse pressure; Ea, effective arterial volume elastance; Ees, left ventricular end-systolic elastance; PNE, prenatal nicotine exposure.

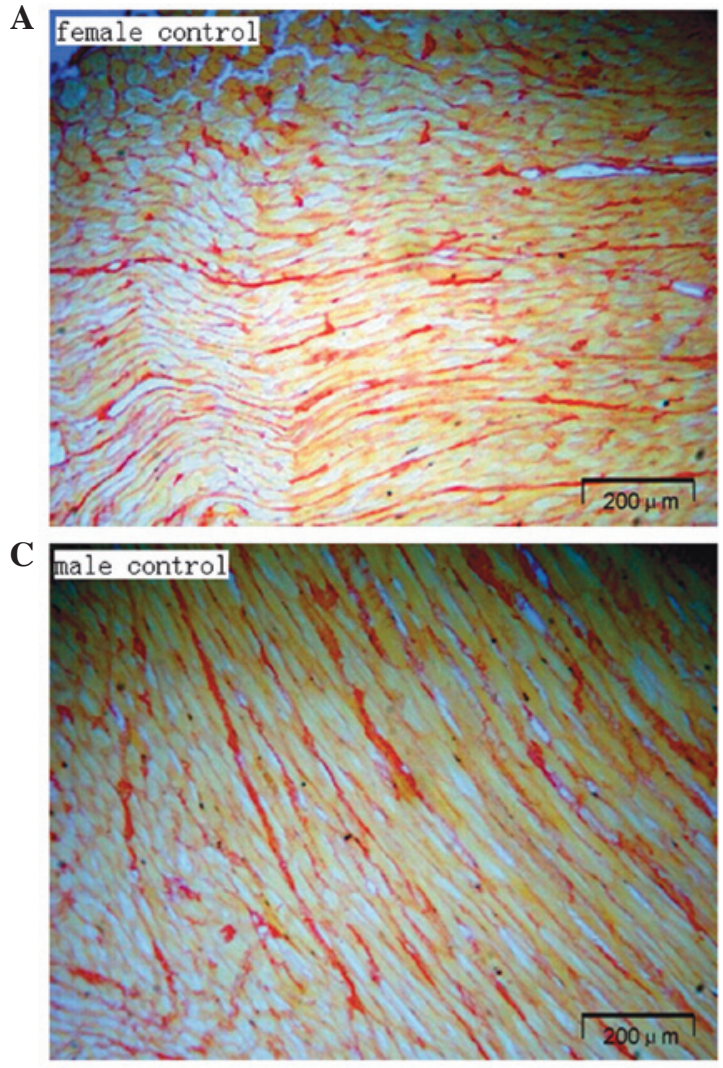

E Collagen content and the percentage of insoluble collagen

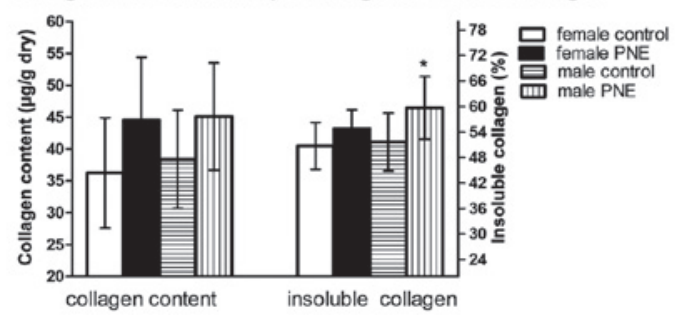

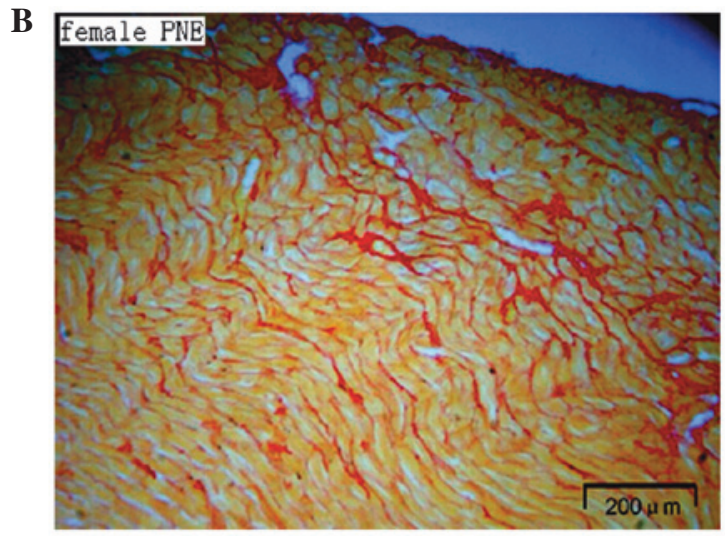

D

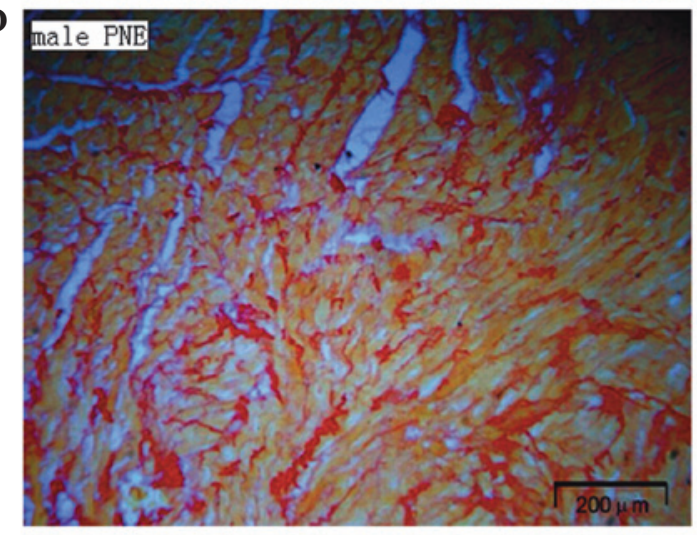

F Transverse cross-sectional area and diameter

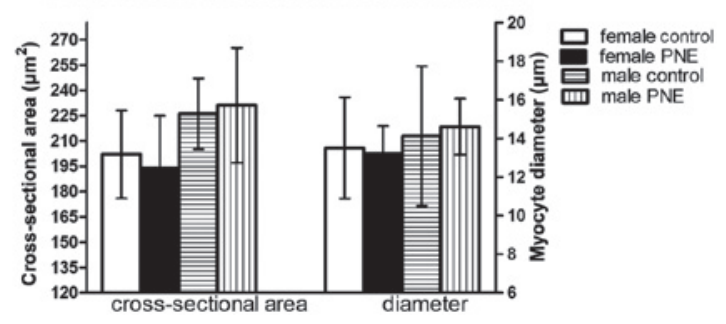

Figure 2. Effect of maternal nicotine administration on left ventricle collagen content (picrosirius red staining) and cardiomyocyte cross-sectional area in left ventricle of adult offspring. The results in the histograms are presented as the mean \pm standard deviation. Representative images of: Female offspring of (A) maternal saline exposure or (B) maternal PNE; and male offspring of (C) maternal saline exposure or (D) maternal PNE. (E) Histogram for total and insoluble collagen content. (F) Histogram for cardiomyocyte cross-sectional area in left ventricle. Values are presented as the mean \pm standard deviation. ${ }^{*} \mathrm{P}<0.05$, versus gender-matched control. PNE, prenatal nicotine exposure. 
A

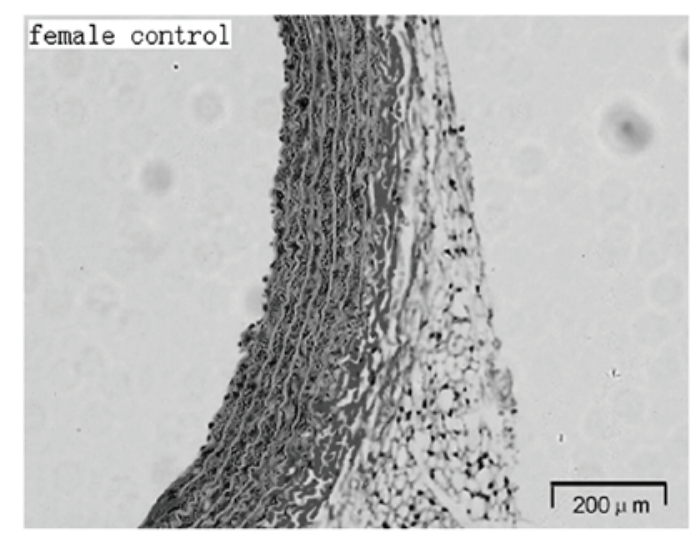

C

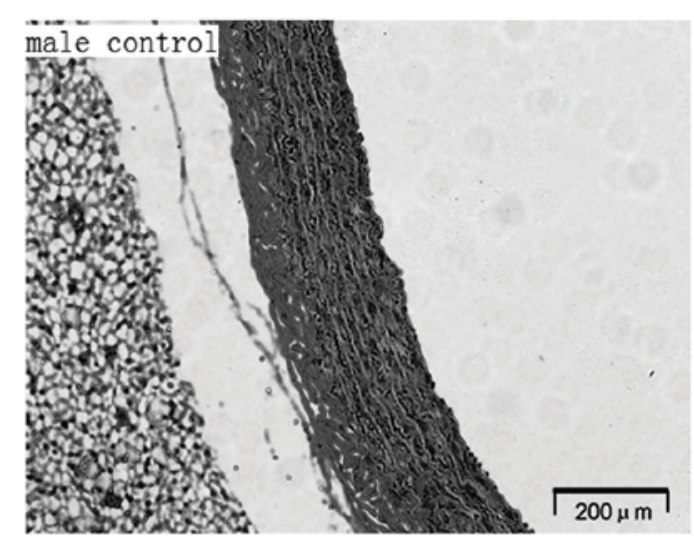

B

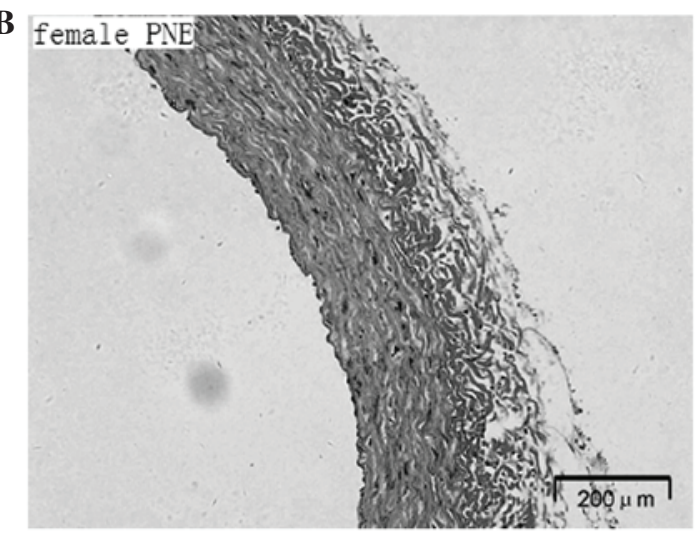

D

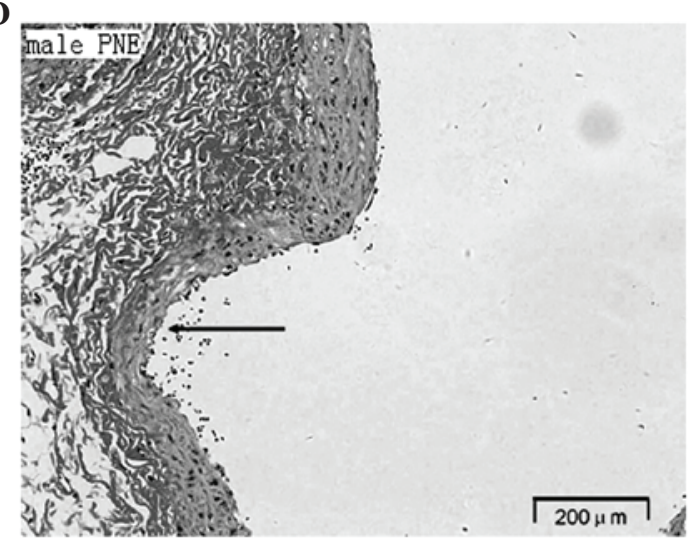

Figure 3. Van Gieson elastin staining revealed decreased elastin density with fractured and disrupted elastin fibers in males and females following maternal nicotine treatment. Representative images of: Female offspring of (A) maternal saline exposure or (B) maternal PNE; and male offspring of (C) maternal saline exposure or (D) maternal PNE Aortic aneurysm occurred due to a localized weakness of the artery (arrow). PNE, prenatal nicotine exposure.

addition, no significant morphological alterations in cardiomyocyte transverse cross-sectional area and diameter were identified among the groups (Fig. 2F).

Elastic properties of the aorta. PNE offspring presented with a significantly lower elastin density as manifested by elastic lamellae (Fig. 3). The arterial media revealed degeneration and fragmentation of the elastic network and disorganization of collagen fibers. Compared with female pups, aortic elastic fibers appeared to be more fractured and disrupted in male offspring. In addition, aortic aneurysm occurred due to a localized weakness of the artery wall.

Medial thickness to internal diameter ratio in the descending aorta and mesenteric artery. In the descending aorta, medial thickness, internal diameter and ratio of medial thickness to internal diameter were comparable between PNE offspring and their gender-matched control rats. Conversely, in resistance-sized mesenteric arteries, the ratio of medial thickness to lumen diameter increased by 35 and $75 \%$ in males and females, respectively (Fig. 4).

\section{Discussion}

The present study revealed the following novel observations, namely, PNE induced ventricular-arterial stiffness characterized with a lower Ea/Ees in males, whereas Ea/Ees was preserved in females. Furthermore, enhanced collagen cross-linking in myocardium, underdeveloped elastic fibers in the aorta and remodeled resistance vessels were noted in male and female pups, with a lesser extent in female offspring. The finding that PNE affected predominantly male offspring is in line with a previous study indicating that male offspring were more sensitive to the manifestation of hypertension caused by adverse prenatal stimuli (26). These results also supported a study demonstrating that PNE altered vascular function, which predisposed male offspring to hypertension during adulthood (27). However, the present data revealed that prenatal nicotine insult modified mesenteric artery medial thickness to internal diameter ratio in male and female offspring, which was somewhat at odds with the observations that mesenteric artery media thickness did not alter significantly in female offspring. A plausible explanation may be that different ages were investigated, 12 months in the present study compared with 5 months in the previous study (15).

Another important finding of the present study was that central systolic pressure and PP demonstrated no significant differences in the male or female pups as compared with their age and gender-matched control. While central diastolic pressure was modestly elevated in PNE offspring, it may represent a compensatory mechanism whereby an increase in pressure in the ascending aorta during diastole may increase coronary blood flow to compensate for shorter diastole.

The present study revealed that Ea as well as AI was elevated following maternal nicotine insult. Ea and AI are primarily an assessment of peripheral resistance and the elastic 


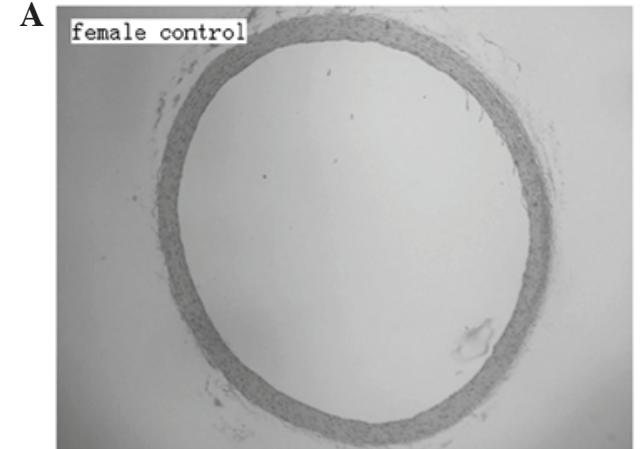

C

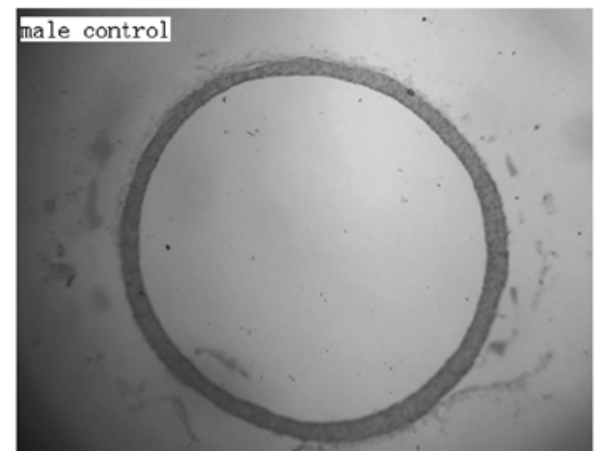

$\mathbf{E}$

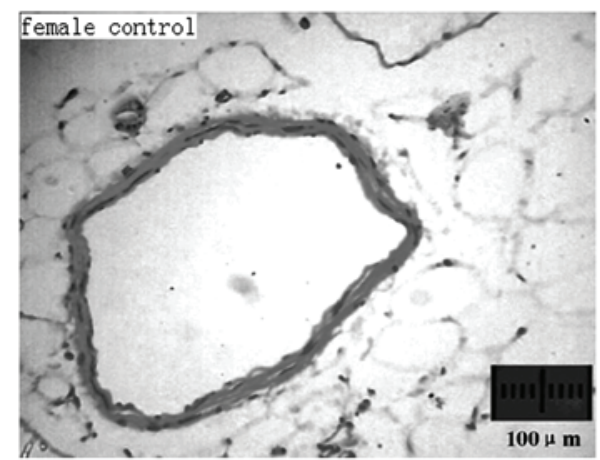

$\mathbf{G}$

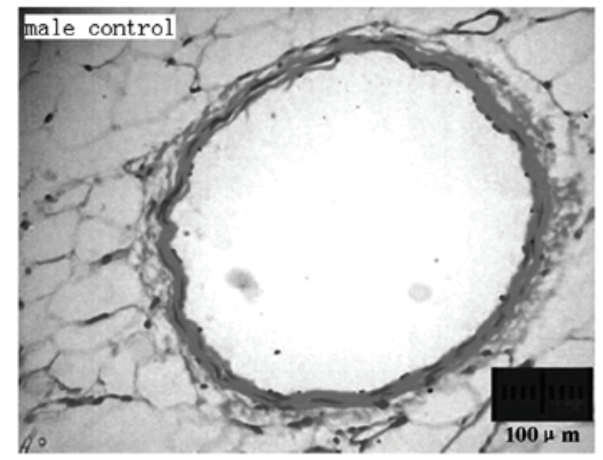

I Medium thickness of aorta and mesenteric artery

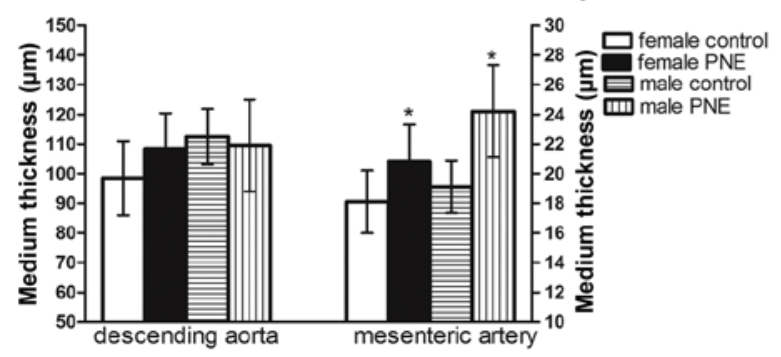

B

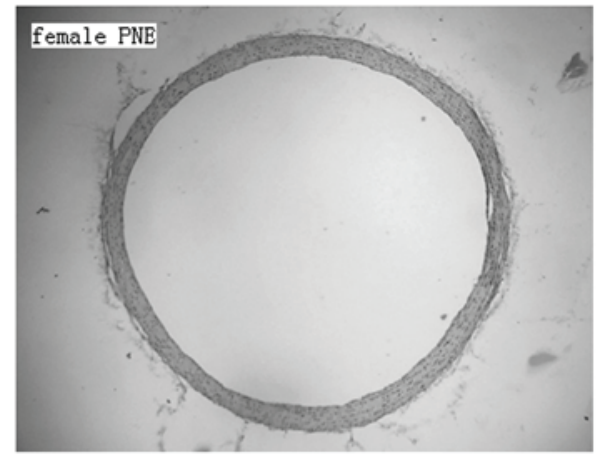

D

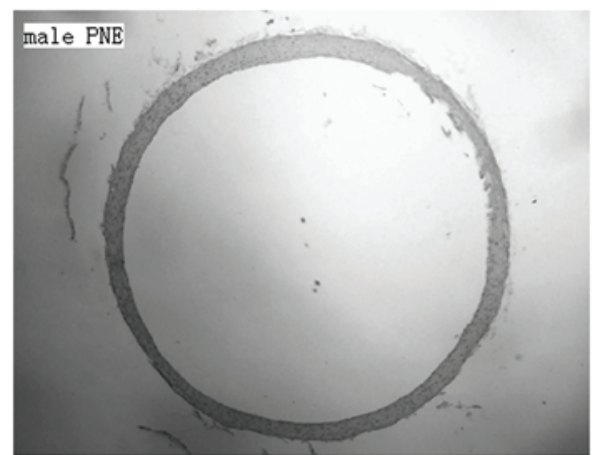

$\mathbf{F}$

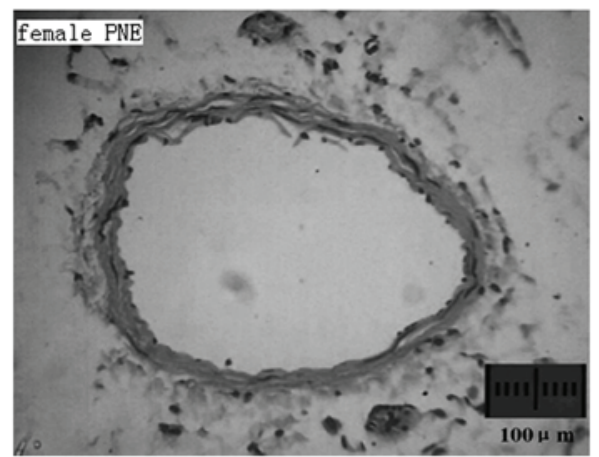

$\mathbf{H}$

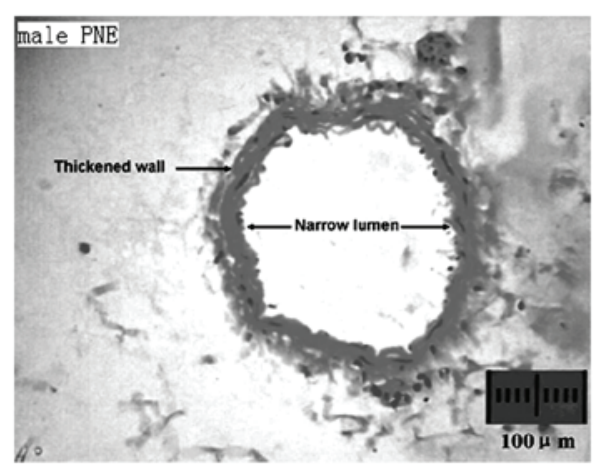

J

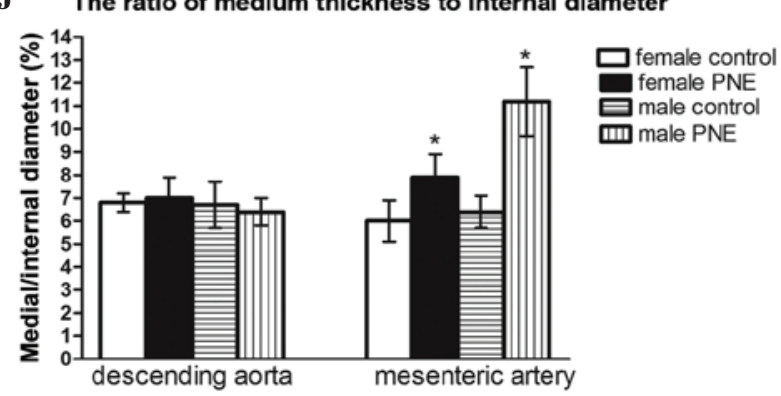

Figure 4. Representative photomicrographs illustrating aorta and resistant vessel medium thickness to internal diameter ratios in offspring exposed to prenatal vehicle or nicotine. Representative images of the descending aorta and resistance-sized mesenteric arteries, respectively, in: Female offspring of (A and E) maternal saline exposure or (B and F) maternal PNE; and male offspring of (C and G) maternal saline exposure or (D and H) maternal PNE Hematoxylin and eosin staining exhibited increased medial thickness to internal diameter ratio in PNE offspring, with more pronounced effects in male pups. (I) Histogram for medial thickness in the aorta and mesenteric artery. (J) Histogram for the ratio of medial thickness to internal diameter in the aorta and mesenteric artery. Values are presented as the mean \pm standard deviation. ${ }^{*} \mathrm{P}<0.05$, versus gender-matched control. PNE, prenatal nicotine exposure. 
properties of large conduit arteries (28). The present data revealed increased fragmentation of the elastic network. There was also an increase in the ratio of medial thickness to internal diameter in mesenteric arteries. These results were in line with a previous study demonstrating that smoking had acute and chronic detrimental effects on ascending aortic elastic properties in healthy male subjects (29). Elevated AI in male PNE offspring implies that peripheral waves arrive earlier. It occurs early in the cardiac cycle during systole and thus results in increased myocardial load and reduced coronary perfusion, eventually compromising cardiac function and structure.

The present observations implied LV myocardium CSA and LV weight/BW were altered insignificantly; however, collagen solubility (an index of collagen cross-linking) was decreased in parallel with increased left ventricular meridional wall stress. These results were substantiated by other studies demonstrating that enhanced collagen cross-linking exacerbated myocardium distensibility without LV hypertrophy $(30,31)$. Ees principally mirrors LV systolic stiffening. Elevated ventricular elastance may augment systolic pressure sensitivity to cardiac loading, increase cardiac energy cost and myocardial oxygen consumption to deliver stroke volume and further impair cardiac structure and function.

Under normal conditions, the Ea/Ees ratio is close to 1 (0.3-1.3) resulting in maximal stroke work and cardiac metabolic efficiency (32). The present results suggested that although cardiovascular stiffness was evident in males and females by 12 months of age, only males exhibited suboptimal arterial-ventricular coupling. These findings may assist in elucidating the reasons that intrauterine insults predisposed male offspring to cardiovascular function disequilibrium (33).

Decreased aortic elasticity concomitant with enhanced peripheral resistance led to increased arterial loading, wave reflection velocity and $\mathrm{AI}$, which in turn resulted in $\mathrm{LV}$ augmented end-systolic stress, modified collagen composition and diminished cardiac performance.

The mechanisms through which PNE affects ventricular-arterial coupling are various. It has been demonstrated that nicotine downregulates miR-133 and miR-590 by the nicotinic acetylcholine receptor, $\alpha 7-n A C h R$, subsequently activates the transforming growth factor (TGF)- $\beta 1$ receptor and increases the synthesis of TGF- $\beta 1$ protein, resulting in structural alterations of increased collagen and reduced elastin content in vascular media (34). Additionally, maternal nicotine administration caused programming of protein kinase $\mathrm{C} \varepsilon$ gene repression through promoter methylation, simultaneously activating the sympathetic nervous system in the fetal heart, which compromised cardiovascular homeostasis (35).

The exact mechanisms underlying the gender dimorphism in nicotine-mediated cardiovascular homeostasis remain to be elucidated. These gender-specific alterations may be directly associated with differences in sensitivity and adaptation to the specific insult while in utero, or females may have greater vascular compensatory mechanisms following birth.

There are, however, several limitations regarding the present study. With reference to the study design, Ea/Ees was not invasively quantified from catheterization-derived pressure-volume loops. However, the validations of noninvasive single-beat determination of left ventricular end-systolic elastance with echocardiography have been published previously (36). Additionally, studies were performed under anesthesia. Future studies that investigate hemodynamics in a conscious animal may be useful to confirm this data. Finally, several studies have suggested collagen phenotype affects myocardial stiffness $(30,37)$. Further studies are required in order to clarify its independent role in determining myocardial stiffness in the present model.

In conclusion, the present data indicated that PNE caused combined ventricular-arterial stiffening in male and female offspring, with lower Ea/Ees in males but preserved Ea/Ees in females. Enhanced collagen cross-linking in myocardium, underdeveloped elastic fibers in the aorta and remodeled resistance vessels were associated with the pathological ventricular arterial mismatching. Higher ventricular and arterial stiffness has important implications for blood pressure instability and loading sensitivity. It is essential for early intervention and the development of treatment strategies to understand how the fetus adapts to an adverse intrauterine environment and how this permanently affects cardiovascular function, particularly prior to the onset of detectable cardiovascular complications. Further studies at a molecular level are encouraged to elucidate the effects of PNE on ventricular and arterial coupling.

\section{Acknowledgements}

This study was supported in part by the National Natural Science Foundation of China (grant no. 81000129), the Natural Science Foundation of Fujian Province (grant nos. 13131037 and 2014J06018), the Scientific Research Foundation for the Returned Overseas Chinese Scholars, State Education Ministry (grant no. 2009-1549), the Foundation from Medical Innovation Project of Fujian Province (grant no. 2011-CX-25) and by the Major Program Foundation of Fujian Medical University (grant no. 09ZD015). The authors would like to thank Mr. Changsheng $\mathrm{Xu}$ and Mr. Liangming Zhang (Fujian Institute of Hypertension) for their technical assistance with histomorphometric analysis. The abstract for the present study has previously been published (http://content.onlinejacc.org/article.aspx?articleID=1913711).

\section{References}

1. Hanna ST: Nicotine effect on cardiovascular system and ion channels. J Cardiovasc Pharmacol 47: 348-358, 2006.

2. Benowitz NL and Gourlay SG: Cardiovascular toxicity of nicotine: implications for nicotine replacement therapy. J Am Coll Cardiol 29: 1422-1431, 1997.

3. Wang Z, Huang Z, Lu G, et al: Hypoxia during pregnancy in rats leads to early morphological changes of atherosclerosis in adult offspring. Am J Physiol Heart Circ Physiol 296: H1321-H1328, 2009.

4. Gao YJ, Holloway AC, Zeng ZH, et al: Prenatal exposure to nicotine causes postnatal obesity and altered perivascular adipose tissue function. Obes Res 13: 687-692, 2005.

5. Rueda-Clausen CF, Morton JS and Davidge ST: Effects of hypoxia-induced intrauterine growth restriction on cardiopulmonary structure and function during adulthood. Cardiovase Res 81: 713-722, 2009

6. Lawrence J, Xiao D, Xue Q, et al: Prenatal nicotine exposure increases heart susceptibility to ischemia/reperfusion injury in adult offspring. J Pharmacol Exp Ther 324: 331-341, 2008.

7. Gao YJ, Holloway AC, Su LY, et al: Effects of fetal and neonatal exposure to nicotine on blood pressure and perivascular adipose tissue function in adult life. Eur J Pharmacol 590: 264-268, 2008.

8. Xiao D, Huang X, Lawrence J, et al: Fetal and neonatal nicotine exposure differentially regulates vascular contractility in adult male and female offspring. J Pharmacol Exp Ther 320: 654-661, 2007. 
9. Kim JW, Park CG, Hong SJ, et al: Acute and chronic effects of cigarette smoking on arterial stiffness. Blood Press 14: 80-85, 2005.

10. Goette A, Lendeckel U, Kuchenbecker A, et al: Cigarette smoking induces atrial fibrosis in humans via nicotine. Heart 3: 1056-1063, 2007.

11. Rajiyah G, Agarwal R, Avendano G, et al: Influence of nicotine on myocardial stiffness and fibrosis during chronic ethanol use. Alcohol Clin Exp Res 20: 985-989, 1996.

12. Fourie PR, Coetzee AR and Bolliger CT: Pulmonary artery compliance: its role in right ventricular-arterial coupling. Cardiovasc Res 26: 839-844, 1992.

13. Boychuk CR and Hayward LF: Prenatal nicotine exposure alters postnatal cardiorespiratory integration in young male but not female rats. Exp Neurol 232: 212-221, 2011.

14. Fennessy F, Casey RG and Bouchier-Hayes D: Peripheral and central arterial haemodynamic interactions are early abnormalities in young male cigarette smokers. Eur J Vasc Endovasc Surg 25: 152-158, 2003.

15. Xiao D, Xu Z, Huang X, et al: Prenatal gender-related nicotine exposure increases blood pressure response to angiotensin II in adult offspring. Hypertension 51: 1239-1247, 2008.

16. Sumitra M, Manikandan P, Rao KV, et al: Cardiorespiratory effects of diazepam-ketamine, xylazine-ketamine and thiopentone anesthesia in male Wistar rats - a comparative analysis. Life Sci 75: 1887-1896, 2004.

17. Jegger D, da Silva R, Jeanrenaud X, et al: Ventricular-arterial coupling in a rat model of reduced arterial compliance provoked by hypervitaminosis D and nicotine. Am J Physiol Heart Circ Physiol 291: H1942-H1951, 2006.

18. Weinberg EO, Thienelt CD, Katz SE, et al: Gender differences in molecular remodeling in pressure overload hypertrophy. J Am Coll Cardiol 34: 264-273, 1999.

19. Sahlén A, Shahgaldi K, AagaardP, etal: Altered ventriculo-arterial coupling during exercise in athletes releasing biomarkers after endurance running. Eur J Appl Physiol 112: 4069-4079, 2012.

20. Blaudszun G and Morel DR: Relevance of the volume-axis intercept, V0, compared with the slope of end-systolic pressure-volume relationship in response to large variations in inotropy and afterload in rats. Exp Physiol 96: 1179-1195, 2011.

21. London GM, Blacher J, Pannier B, et al: Arterial wave reflections and survival in end-stage renal failure. Hypertension 38: 434-438, 2001.

22. Lartaud I, Gaillard V, Dauca M, et al: Pioglitazone protects against elastocalcinosis and improves aortic wall elasticity. Ann Pharm Fr 65: 189-194, 2007 (In French).

23. Leipner C, Grün K, Müller A, et al: Imatinib mesylate attenuates fibrosis in coxsackievirus b3-induced chronic myocarditis Cardiovasc Res 79: 118-126, 2008.
24. Marque V, Kieffer P, Gayraud B, et al: Aortic wall mechanics and composition in a transgenic mouse model of Marfan syndrome. Arterioscler Thromb Vasc Biol 21: 1184-1189, 2001.

25. Yamamoto K, Masuyama T, Sakata Y, et al: Myocardial stiffness is determined by ventricular fibrosis, but not by compensatory or excessive hypertrophy in hypertensive heart. Cardiovasc Res 55: 76-82, 2002.

26. do Carmo Pinho Franco M, Nigro D, Fortes ZB, et al: Intrauterine undernutrition - renal and vascular origin of hypertension. Cardiovasc Res 60: 228-234, 2003.

27. Xiao D, Huang X, Yang S and Zhang L: Estrogen normalizes perinatal nicotine-induced hypertensive responses in adult female rat offspring. Hypertension 61: 1246-1254, 2013.

28. Heffernan KS, Patvardhan EA, Hession M, et al: Elevated augmentation index derived from peripheral arterial tonometry is associated with abnormal ventricular-vascular coupling. Clin Physiol Funct Imaging 30: 313-317, 2010.

29. Sassalos K, Vlachopoulos C, Alexopoulos N, et al: The acute and chronic effect of cigarette smoking on the elastic properties of the ascending aorta in healthy male subjects. Hellenic J Cardiol 47: 263-268, 2006.

30. Norton GR, Tsotetsi J, Trifunovic B, et al: Myocardial stiffness is attributed to alterations in cross-linked collagen rather than total collagen or phenotypes in spontaneously hypertensive rats. Circulation 96: 1991-1998, 1997.

31. Antonini-Canterin F, Carerj S, Di Bello V, et al: Arterial stiffness and ventricular stiffness: a couple of diseases or a coupling disease? A review from the cardiologist's point of view. Eur J Echocardiogr 10: 36-43, 2009.

32. Frenneaux M and Williams L: Ventricular-arterial and ventricular-ventricular interactions and their relevance to diastolic filling. Prog Cardiovasc Dis 49: 252-262, 2007.

33. Xue Q and Zhang L: Prenatal hypoxia causes a sex-dependent increase in heart susceptibility to ischemia and reperfusion injury in adult male offspring: role of protein kinase $\mathrm{C}$ epsilon. J Pharmacol Exp Ther 330: 624-632, 2009.

34. Goette A: Nicotine, atrial fibrosis and atrial fibrillation: do microRNAs help to clear the smoke? Cardiovasc Res 83: 421-422, 2009

35. Lawrence J, Chen M, Xiong F, et al: Foetal nicotine exposure causes $\mathrm{PKC} \varepsilon$ gene repression by promoter methylation in rat hearts. Cardiovasc Res 89: 89-97, 2011.

36. Chen $\mathrm{CH}$, Fetics B, Nevo E, et al: Noninvasive single-beat determination of left ventricular end-systolic elastance in humans. J Am Coll Cardiol 38: 2028-2034, 2001

37. Kato S, Spinale FG, Tanaka R, et al: Inhibition of collagen crosslinking: effects on fibrillar collagen and ventricular diastolic function. Am J Physiol 269 (3 Pt 2): H863-H868, 1995. 